\title{
Research on Power Generation Energy Sources Structure Adjustment Algorithm Based on HyperGraph
}

\author{
Chunhua Qiu ${ }^{1, ~}$, Shaoyun $\mathrm{Ge}^{1}$, Ting Yang $^{1}$, Jun Wei ${ }^{2}$, Guoxing Xiang ${ }^{3}$ \\ ${ }^{1}$ Department of Electrical Engineering, Tianjin University, Tianjin, China \\ ${ }^{2}$ Mining Department, Guizhou University, Guiyang, China \\ ${ }^{3}$ Department of Hydropower Engineering, Wuhan University, Wuhan, China \\ Email address: \\ 737267494@qq.com (Chunhua Qiu), faith2003cngz@163.com (Chunhua Qiu),syge@tju.edu.cn (Shaoyun Ge), \\ yangting@tju.edu.cn (Ting Yang),790187947@qq.com (Jun Wei),514819770@qq.com (Guoxing Xiang) \\ ${ }^{*}$ Corresponding author
}

To cite this article:

Chunhua Qiu, Shaoyun Ge, Ting Yang, Jun Wei, Guoxing Xiang. Research on Power Generation Energy Sources Structure Adjustment Algorithm Based on HyperGraph. American Journal of Energy Engineering. Vol. 7, No. 2, 2019, pp. 49-54. doi: 10.11648/j.ajee.20190702.12

Received: June 5, 2019; Accepted: June 29, 2019; Published: July 10, 2019

\begin{abstract}
Nowadays, the energy structure is gradually changing to clean power generation. Clean energy mainly includes renewable energy and part of non-renewable energy. Non-renewable energy is depleting day by day, showing a shrinking trend. Renewable energy is not affected by energy shortage, and is the focus of future development. How to ensure the sustainable and healthy development of clean energy, it is necessary to adjust the existing power generation energy structure scientifically and rationally. In this paper, the theory of hypergraph is introduced to cluster the optimal combination information of clean energy, and a hypergraph model of power generation energy structure adjustment is established. The problem of replacing fossil energy in power generation energy consumption with clean energy is solved as the original objective. By mapping the generation energy structure adjustment with hypergraph, the problem of generation energy structure adjustment is transformed into the problem of solving hypergraph path. By using the two-point hyperpath algorithm, an optimal path for the development of clean power generation, reducing the proportion of fossil energy power generation, and gradually converting to clean energy is obtained. The application of hypergraph algorithm in the structural adjustment of power generation is of great significance to promote the diversification of power generation energy, especially in the clean development, low-carbon development and green development of the power industry.
\end{abstract}

Keywords: Hyper Graph, Power Generation Energy, Restructuring, Algorithm

\section{Introduction}

In the past 10 years, since 2007, clean energy, especially renewable energy, has developed rapidly in most countries of the world, and the proportion of renewable energy in power generation has increased year by year. The proportion of installed wind and solar power in the United States has increased from less than $1 \%$ to $7.5 \%[1,2]$; in Europe, the proportion has increased from $3 \%$ to more than $13 \%$ [3], and the proportion of wind and solar power has also increased from $1 \%$ to $5 \%$ in China. At present, clean energy is one of the important contents of the 13th Five-Year Plan of Electric Power Development in China. It has been made clear that priority should be given to the distribution of clean energy in the adjustment of electric power structure, that is, to develop new energy in the balance of electric power. It is expected that by 2020 , the proportion of coal in China's energy structure will fall below $57 \%$, renewable energy will increase to about $10 \%$, and the installed capacity of non-fossil energy power generation is expected to reach 770 million kilowatts [4]. By 2050 , China will achieve the goal that $60 \%$ of its electricity will come from renewable energy sources $[5,6]$. This requires us to coordinate the development of clean energy scientifically and reasonably, and to develop renewable energy in an orderly manner, in accordance with the principle of combining centralized development with decentralized development and focusing on near absorption.

How to optimize and adjust the energy structure of power 
generation, from the perspective of combination planning, is to solve the target problem of clean power generation in a specific environment. As one of the effective modeling tools for combinatorial planning problems, HyperGraph theory is an important branch of graph theory. The above problems can be regarded as the target function to be realized. The entity objects related to the energy structure of power generation can be regarded as vertices, and the relationship between them can be regarded as supersides to build mathematical models. The process of power generation energy structure adjustment is to find out the possible development path between entities, judge whether various entities are related, find out the possible hypergraph path and make the corresponding semantic mapping, and then make a good explanation of its optimal combination scheme.

\section{Hypergraph Theory and Its Algorithms}

\subsection{Relevant Definitions of Hypergraphs}

In the 1970 s, French mathematician c. erge proposed the hypergraph theory. Compared with the classical network graph theory, which only considers the network topology composed of two-terminal components and four-terminal controlled sources, hypergraph studies the hypernetwork topology composed of multi-terminal networks [7, 8]. The main difference between hypergraph and general graph is that one hyperedge can connect multiple points. As a new applied research topic, hypergraph theory is attracting more and more attention.

Definition 1: let $\mathrm{V}=\{\mathrm{v} 1, \mathrm{v} 2 \ldots, \mathrm{vn}\}$ is a finite set, and the hypergraph $\mathrm{H}$ on $\mathrm{V}$ is a subset of $\mathrm{V}, \mathrm{H}=(\mathrm{E} 1, \mathrm{E} 2 \ldots, \mathrm{Em})$ meet:

$$
\begin{gathered}
\mathrm{Ei} \neq \phi \quad(\mathrm{i}=1,2, \ldots, \mathrm{m}) \\
\bigcup_{i=1}^{m} E_{i}=V
\end{gathered}
$$

If it is satisfied, then $\mathrm{H}$ is called simple hypergraph [9, 10]. In the hypergraph $\mathrm{H}$, the elements of $\mathrm{V}, \mathrm{v} 1, \mathrm{v} 2 \ldots$, vn is called vertex, set E1, E2... Em is called edge.

Definition 2: hypergraph $\mathrm{H}$ is called connected hypergraph if at least one hyperpath exists at any two points of hypergraph $\mathrm{H}$. On the contrary, if there is no hyperpath between at least two points in the hypergraph $\mathrm{H}$, the hypergraph $\mathrm{H}$ is a disconnected hypergraph [5].

Definition 3: for the hypergraph $\mathrm{H}=(\mathrm{V}, \mathrm{E})$, if there is a subhypergraph $\mathrm{Ht}=(\mathrm{V} ; \mathrm{E} 1, \mathrm{E} 2, \ldots, \mathrm{Et})$ is a connected hypergraph without hyperloop, then subhypergraph $\mathrm{Ht}$ is called hypertree. If $\mathrm{H}$ is a nonconnected hypergraph, then $\mathrm{Ht}$ is called a hyperforest $[11,12]$.

In the optimal combination hypergraph model of clean energy, all kinds of clean energy and users are abstracted as vertices, and the relationship between vertices is abstracted as edges (hyperedges). The weight given by each edge represents the correlation strength. The optimal combination information of clean energy is clustered and analyzed, and the hypergraph model is established.

\subsection{Hypergraph Path Solving Algorithms}

The key to solve the problem with hypergraph theory is to solve the hypergraph path. Hypergraph path solving algorithms are different, and the operation workload and complexity are quite different. Currently, there are mainly hypergraph generating hypertree algorithm, dijkstra labeling method, Floyd algorithm and two-point hyperpath solving algorithm. The hypergraph generation hypertree algorithm and the two-point hyperpath solution algorithm both search and traverse the construction tree through the construction tree to obtain the path. However, the hypergraph generation hypertree algorithm can only solve the birth hypertree when the hypergraph is connected with the hypergraph, and the traversal result includes at most a hyperpath from the origin to the destination. Relatively speaking, the two-point hyperpath solution algorithm does not require the hypergraph to be only connected hypergraph, and multiple hyperpaths between the origin and the destination can be obtained [13].

Using dijkstra labeling method to solve the shortest path between any two points, each vertex needs to be calculated separately, and the distance matrix between each vertex is obtained. And more desirably, using Floyd hypergraph shortest path algorithm, empowerment matrix as the distance matrix of the initial value, the distance matrix by means of vertex insertion of iteration, the matrix can be obtained at the same time, the distance matrix and the insertion point matrix, again according to the query to the shortest distance insertion point matrix corresponds to the shortest path, greatly reduce the computational complexity [14-16]. This paper is mainly based on Floyd algorithm to solve the optimization hypergraph of the comprehensive development of clean energy. The shortest path matrix between each two points of the hypergraph is obtained through the weight matrix of the hypergraph. The state transfer equation is: map $[I, \mathrm{j}]:=\min \{\operatorname{map}[\mathrm{I}, \mathrm{j}]+\operatorname{map}[\mathrm{k}, \mathrm{j}]$, map $[\mathrm{I}, \mathrm{j}]$, where map $[I, j]$ represents the shortest distance from $I$ to $j$, and $\mathrm{k}$ is the endpoint of exhaustive $\mathrm{I}, \mathrm{j}$. The basic process of Floyd algorithm is as follows: starting from any unilateral path, the distance between all two points is the edge weight; if there is no edge connection between two points, the weight is infinite. For each pair of vertices I and $\mathrm{j}$, determine if there is a vertex $\mathrm{k}$ that makes the path from $\mathrm{I}$ to $\mathrm{k}$ to $\mathrm{j}$ shorter than the one you know, and if there is, update it. The procedure of calculation example is shown in Figure 1 and the following calculation.

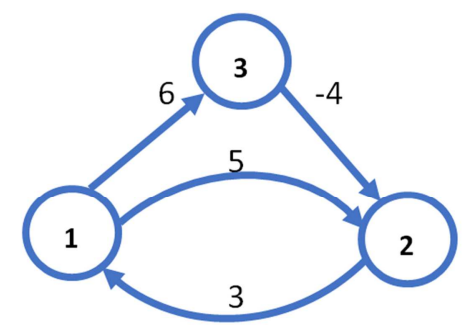

D matrix

P matrix

Figure 1. Floyd algorithm. 
Table 1. Distance matrix $D^{0}$.

\begin{tabular}{lllll}
\hline & & 1 & 2 & 3 \\
$\mathrm{D}^{0}=$ & 1 & 0 & 5 & 6 \\
& 2 & 3 & 0 & $\infty$ \\
& 3 & $\infty$ & -4 & 0 \\
\hline
\end{tabular}

Table 2. Insertion point matrix $P$.

\begin{tabular}{lllll}
\hline & & 1 & 2 & 3 \\
$\mathrm{P}=$ & 0 & 0 & 0 \\
& 1 & 0 & 0 & 0 \\
& 2 & 0 & 0 & 0 \\
\hline
\end{tabular}

$\mathrm{k}=1$,

Table 3. Distance matrix $D^{l}$

\begin{tabular}{lllll}
\hline \multirow{3}{*}{$\mathrm{D}^{1}=$} & & 1 & 2 & 3 \\
& 1 & 0 & 5 & 6 \\
& 2 & 3 & 0 & 9 \\
& 3 & $\infty$ & -4 & 0 \\
\hline
\end{tabular}

Table 4. Insertion point matrix $P$.

\begin{tabular}{lllll}
\hline & & 1 & 2 & 3 \\
$\mathrm{P}=$ & 0 & 0 & 0 \\
& 1 & 0 & 0 & 1 \\
& 2 & 0 & 0 & 0 \\
\hline
\end{tabular}

$\mathrm{D} 1[2,3]=\min (\mathrm{D} 0[2,3], \mathrm{D} 0[2,1]+\mathrm{D} 0[1,3])=\min (\infty, 9)=9$. $\mathrm{D} 1[3,2]=\min (\mathrm{D} 0[3,2], \mathrm{D} 0[3,1]+\mathrm{D} 0[1,2])=\min (-4, \infty)=-4$.

$\mathrm{k}=2$,

Table 5. Distance matrix $D^{2}$

\begin{tabular}{lllll}
\hline \multirow{3}{*}{$\mathrm{D}^{2}=$} & 1 & 2 & 3 \\
& 1 & 0 & 5 & 6 \\
& 2 & 3 & 0 & 9 \\
& 3 & -1 & -4 & 0 \\
\hline
\end{tabular}

Table 6. Insertion point matrix $P$

\begin{tabular}{lllll}
\hline & & 1 & 2 & 3 \\
$\mathrm{P}=$ & 1 & 0 & 0 & 0 \\
& 2 & 0 & 0 & 1 \\
& 3 & 2 & 0 & 0 \\
\hline
\end{tabular}

$\mathrm{k}=3$,

Table 7. Distance matrix $D^{3}$.

\begin{tabular}{lllll}
\hline \multirow{3}{*}{$=$} & & 1 & 2 & 3 \\
$\mathrm{D}^{3}=$ & 1 & 0 & 2 & 6 \\
& 2 & 3 & 0 & 9 \\
& 3 & -1 & -4 & 0 \\
\hline
\end{tabular}

Table 8. Insertion point matrix $P$.

\begin{tabular}{lllll}
\hline & & 1 & 2 & 3 \\
$\mathrm{P}=$ & 0 & 3 & 0 \\
& 1 & 0 & 0 & 1 \\
& 2 & 2 & 0 & 0 \\
\hline
\end{tabular}

$\mathrm{D} 3[1,2]=\min (\mathrm{D} 2[1,2], \mathrm{D} 2[1,3]+\mathrm{D} 2[3,2])=\min (5,6-4)=2$.

$\mathrm{D} 3[2,1]=\min (\mathrm{D} 2[2,1], \mathrm{D} 2[2,3]+\mathrm{D} 2[3,1])=\min (3,9-1)=3$.

From the above, Floyd algorithm will select a vertex each time, traverse the entire matrix, find the value that needs to be updated, and find the shortest path between vertices in the hypergraph through multiple iterations.

\section{Research on Algorithms of Hypergraph in Power Supply Structure Adjustment}

\subsection{Definition and Types of Clean Energy}

Energy is the material basis of human activities, and the development of energy is the common concern of the world. The development of human society can not be separated from the emergence of high-quality energy and the use of advanced energy technologies. The purpose of power generation energy structure adjustment is to use clean energy technology to optimize power generation energy structure, make the proportion of energy more reasonable, and improve the overall efficiency of energy development and utilization. Clean energy is green energy, which is environmentally friendly and belongs to high-quality energy. Clean energy is a kind of energy with advanced conversion efficiency and good economy, and low or no pollution to the ecological environment in the whole process of development and utilization, including its production and consumption [17-20].

Clean energy mainly includes renewable energy and some non-renewable energy. Among them, renewable energy can be recycled and sustained in nature, such as water, wind, solar, biomass, tidal and geothermal energy, which can be restored and supplemented after consumption. Some non-renewable energy belonging to clean energy refers to nuclear energy and natural gas. Because fossil energy is limited and increases the risk of greenhouse effect, renewable energy will play an increasingly important role with the shortage of earth resources.

\subsection{Necessity and Main Problems of Clean Power Generation}

In today's world, with the rapid development of global economy, economic development can not be separated from all kinds of energy. With the increasing demand for energy and the strengthening of environmental protection in the world, the development of clean energy has become an inevitable trend. At present, the main problems in the development of clean energy in China are mainly manifested in the following three aspects:

i. The annual utilization hours of power generation are low, and the generating efficiency of generating units is not high. Take wind power projects as an example, the inland areas with abundant wind energy resources are mainly concentrated in the grasslands and Gobi beaches in the north, northwest and northeast of China, where the electricity load is very small, the annual utilization hours of wind turbines are low, and the power generation efficiency is not high. In China, the electricity load is mainly concentrated in the central and Eastern regions. The distribution of electricity load 
center and energy capacity is inverse, and it is difficult to absorb wind power in situ.

ii. The cost of power generation is on the high side, and the technical level needs to be improved. Taking wind power projects as an example, the eastern coastal areas and islands of China are rich in wind energy resources, but the construction technology of offshore wind power is difficult, the construction of supporting power grids is relatively lagging behind, and the transportation of grasslands, Gobi beaches and islands is inconvenient, the proportion of wind turbines in the initial investment of wind farms is large, the transportation of equipment is difficult, and the project development cost is high.

iii. Economic returns are low, and investment and operation still need government subsidies. The state's support policy for renewable energy is necessary and administrative in the early stage of development. At present, in provinces rich in wind energy, users need to pay more electricity prices, which will cause a great burden to provinces and autonomous regions with poor affordability of electricity prices. In addition, due to the volatility and intermittence of wind power, it is necessary to build peak-shaving power supply for grid-connected. The single structure of power supply in North China, Northeast China and Northwest China is a severe disaster area of wind abandonment and power limitation. Some wind and photovoltaic power enterprises have encountered difficulties in capital turnover and losses. If we continue to use the idea of subsidy, the subsidy gap will expand to more than 20 billion yuan by 2020 , which is not conducive to the sustained and healthy development of the economy.

Therefore, it is necessary to take measures according to local conditions, actively and steadily promote the structural adjustment of power generation energy, establish safeguard measures for clean energy development, and promote the demonstration effect of clean power generation.

\subsection{Superpath Algorithm for Power Generation Energy Structure Adjustment}

\subsubsection{Hypergraph Mapping and Its Model}

The problem of energy structure adjustment in power generation enterprises is modeled by using hypergraph model. The original objective problem is to replace fossil energy in power generation energy consumption with clean energy. The hyperpath between the two vertices is the solution of the objective problem. The hypergraph mapping of power generation energy structure adjustment is shown in Table 9. The hypergraph model of power generation energy structure adjustment is shown in Figure 2.

Table 9. Hypergraph Model Mapping for Optimal Adjustment of Power Generation Energy Structure.

\begin{tabular}{llll}
\hline The model definition & Data unit & Semantic mapping & Mathematical symbols \\
\hline Vertex & Object & Substantive Object of Power Generation Energy Structure Adjustment \\
Super Edge & Relationship & Relatively Independent Functional Units of Power Generation Enterprises $\quad$ e1, e2,..., em \\
Hypergraph & Aggregation & Structural Adjustment and Combination of Power Generation Energy & (V, E) \\
\hline
\end{tabular}

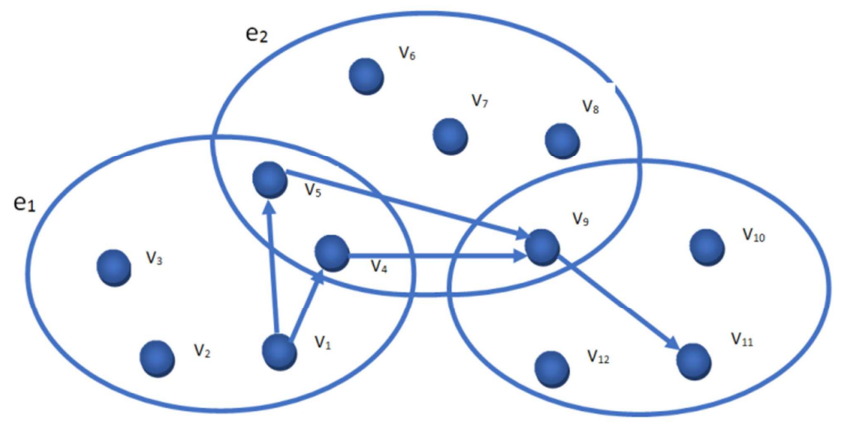

$\mathrm{e}_{3}$

Figure 2. Generation Energy Structure Adjustment Hypergraph Model.

According to the abstract hypergraph model of power generation structure adjustment in Figure 3, the corresponding bipartite graph $\mathrm{G}<\mathrm{H}>$ is shown in Figure 3 .

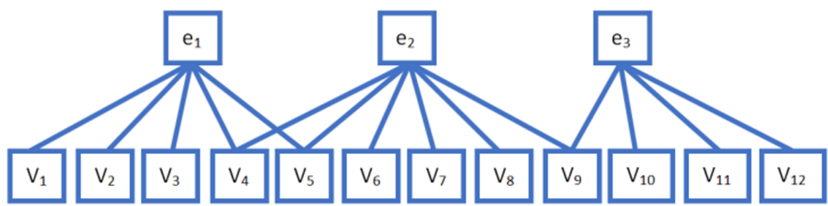

Figure 3. Bipartite graph $G<H>$.

\subsubsection{Hyperpath Solution}

The hyperpath algorithm based on two points is used to solve the hyperpath from vertex V1 to V11. The relation matrix corresponding to the bipartite graph $\mathrm{G}<\mathrm{H}>$ is shown in Figure 4.

$\left.\mathrm{A}=\begin{array}{c|c|c|c|c|c|c|c|c|c|c|c|c|c|c|}\hline & v_{1} & v_{2} & v_{3} & v_{4} & v_{5} & v_{6} & v_{7} & v_{8} & v_{9} & v_{10} & v_{11} & v_{12} \\ \hline & e_{1} & 1 & 1 & 1 & 1 & 0 & 0 & 0 & 0 & 0 & 0 & 0 \\ \hline 0 & 0 & 0 & 1 & 1 & 1 & 1 & 1 & 1 & 0 & 0 & 0 \\ \hline & e_{2} & e_{3}\end{array}\right]$

Figure 4. Relation matrix.

Vertex attributes are defined as follows:

V1: Fossil energy (coal, oil and gas).

V2: Fossil energy power generation equipment (steam turbines, diesel engines and gas turbines, etc.).

V3: Greenhouse effect, environmental governance.

V4: Generation cost, utilization efficiency.

V5: On-grid price.

V6: Energy saving and emission reduction standard.

V7: Access to power system, power grid supporting facilities, transmission lines.

V8: Transportation. V9: Electric load. V10: Financial subsidies.

V11: Clean energy (solar, wind, hydro, geothermal, tidal 
and nuclear, etc.).

V12: Clean energy power generation equipment (such as wind turbines, photovoltaics and hydrogenerator sets, etc.).

The vertex path between V1 and V11: i. V1 --- V4 --- V9 --V11; ii. V1 --- V5 --- V9 --- V11.

\subsubsection{Semantic Analysis of Hyperpath for Power Generation Energy Structure Adjustment}

From the above, we can see that there are two paths to find V1 to V11 in hypergraph. One of them is $\{\mathrm{v} 1, \mathrm{e} 1, \mathrm{v} 4, \mathrm{e} 2, \mathrm{v} 9$, $\mathrm{e} 3, \mathrm{v} 11\}$. The semantic interpretation of the hyperpath is as follows: The transformation of fossil energy to clean energy in power generation enterprises is based on the fact that fossil energy (V1) meets the generating conditions of generating units (e1), reforms the energy composition, reduces the cost of generating electricity, improves the utilization rate of electric energy (V4), meets the transmission and distribution demand of power system (e2), expands the consumption of electric load (v9), and responds to the call of "green environmental protection, low carbon energy conservation" of the country. In order to achieve clean power generation as the development direction (e3), reduce the proportion of fossil energy power generation, and gradually complete the conversion to clean energy (v11).

Another superpath from V1 to V11 is $\{\mathrm{v} 1, \mathrm{e} 1, \mathrm{v} 5, \mathrm{e} 2, \mathrm{v} 9, \mathrm{e} 3$, $\mathrm{v} 11]$. The main difference from path 1 is to reduce the price of fossil energy on-line electricity, rationally adjust the price of new energy on-line electricity (V5), stimulate users'willingness to accept clean energy actively in areas rich in new energy such as wind and solar energy, and to absorb on-site load electricity (v9), so as to stimulate the structure of power generation from fossil energy. Conversion of source (V1) to clean energy (v11).

Because lowering the price of fossil energy on the grid will have a great impact on the coal market, and directly related to the economic benefits of power generation enterprises, and is not conducive to the stability of staff, it is necessary to balance the interests of all parties; on the other hand, the current users'willingness to take the initiative to accept clean energy is not strong, and the phenomenon of abandoning wind and light in some areas is serious. Relatively speaking, Path One is more competitive.

\section{Conclusion}

Clean energy is the future direction of energy development. To build a sustainable energy future, on the one hand, renewable energy power generation needs to be vigorously developed, on the other hand, fossil energy clean power generation needs to be promoted. Under the premise of protecting the ecological environment, priority should be given to the development of hydropower; under the premise of ensuring safety, nuclear power should be developed efficiently; wind and solar energy should be vigorously developed as new energy generation according to local conditions, and power supply structure should be continuously optimized to promote clean development, low-carbon development and green development of the power industry [21-23].

This paper attempts to introduce hypergraph theory into the structural adjustment of power generation energy in China. Through the establishment of hypergraph model, this paper introduces the hyperpath solving algorithm, makes quantitative analysis and verification of the application of the hyperpath solving algorithm in the structural adjustment of power generation by empirical research, and then makes qualitative induction on the basis of this, determines the optimal development path from the perspective of combinatorial planning, realizes the combination of theory and empirical research, which is more in line with the development law of things and human beings'overall interests. Based on the rigorous graph theory knowledge, the semantic mapping of hypergraph model and hyperpath in the structural adjustment of power generation, the algorithm obtains a reasonable path which is more conducive to the conversion of fossil energy to clean energy, and makes a new exploration for the application of hypergraph in this field.

\section{References}

[1] James McCalley, Jay Caspary, Christopher Clack, Wayne Galli, Melinda Marquis, Dale Osborn, Antje Orths, Justin Sharp, Vera Silva, and Peter Zeng, "Wide-Area Planning of Electric Infrastructure," IEEE power \& energy magazine, Vol. 15, No. 6, pp. 83-93, November/December 2017

[2] A. MacDonald, C. Clack, A. Alexander, A. Dunbar, J. Wilczak, and Y. Xie, "Future cost-competitive electricity systems and their impact on US CO2 emissions," Nat. Climate Change, vol. 6, pp. 526-531, Jan. 2016.

[3] ENTSO-E. (2016). Ten year network development plan 2016 executive report, European Network of Transmission System perators for Electricity. [Online]. Available: http://tyndp.entsoe.eu/projects/2016-12-20-1600-exec-report.p df.

[4] Yuzhou Zhang, "Strategic Research and Development Strategy of Clean Energy in China," Bulletin of Chinese Academy of Sciences, Vol. 29, No. 4, pp. 429-436, 2014.

[5] Chenhui Tang, Fan Zhang, Ning Zhang, Haoyuan Qu, Li Ma, "Day-ahead Economic Dipatch of Power System Considering Renewable Power Uncertainty and Demand Response," Automation of Electric Power Systems, vol. 43, No. 6, pp. 1-9, May. 2019.

[6] Energy Research Institute of National Development and Reform Commission of the PRC. (2018). China 2050 high renewable energy penetrantion scenario and roadmap study. [Online]. http://news.bjx.com.cn/html/20160608/740762.shtml.

[7] Xiaoman Xu, Yugeng Sun, Shan Yang, Ruji Huang, "Hypergraph theory and its application," Acta Electronica Sinica, Vol. 22, No. 8, pp. 22-71, 1994.

[8] Ke Zhang, Haixing Zhao, Zhonglin Ye, Yu Zhu, "Analysis for all-terminal reliability of hypernetworks," Application Research of Computers, Vol. 37, No. 2, pp. 2-7, 2018.

[9] Miaolin Ye, Spectral Method in Graph and Hypergraph Theory, Anhui University, Hefei, China, p. 36, 2010. 
[10] Jianfeng Pei, The super edge-connectivity and restricted edge-connectivity of hypergraphs, Shanxi University, Taiyuan, China, p. 1, 2018.

[11] Quansheng Cheng, Hypergraph Path Solving Algorithms and Their Applications, Huazhong University of Science and Technology, Wuhan, China, pp. 10-29, 2008.

[12] Jing Yang, Zhang-Bing Zhou, Zhi-Yong Liu, "Sub-hypergraph matching based on adjacency tensor," Computer Vision and Image Understanding, vol. 183, pp. 1-10, June 2019.

[13] Bo Yang, Research on Matrix Algorithm of Simple Graph Problem, Huazhong University of Science and Technology, Wuhan City, China, pp. 2-6, 2017.

[14] Weiji Zhao, Zhanyu Gong, Wen Wang, Shoufang Fan, "Comparison and Analysis of Several Classical Shortest Path Algorithms," Journal of Chifeng (Natural Science Edition), Vol. 34, No. 12, pp. 47-49, 2018.

[15] Ying Ma, Zhilong Chen, He Liu, Tongxing Zhao, "Study on the shortest path selection based on Floyd improved acceleration algorithm," Information Technology and Network Security, Vol. 37, No. 6, pp. 72-75+107, 2018.

[16] Wei-Wei Yuan, "An Algorithm based on Feasiable Path the shortest Path,"Journal of Mudanjiang Normal University, No. 2, pp. 36-37, 2017.

[17] Jiahuan Guan, Problems and Countermeasures in Clean
Energy Construction: A Case Study of China General Nuclear Power Corporation (CGN), Liaoning Normal University, Dalian, China, p. 9, 2018.

[18] Wen Dai, An Empirical Study on the Relationship between Clean Energy and Economic Growth: A Case Study of Guangdong Province, Guangzhou University, Guangzhou, China, p. 8-15, 2018.

[19] Jinxin, Luo, "Duality of Clean Energy Power Generation Technology," Style of Science and Technology, No. 13, p. 179, 2018.

[20] Caineng Zou, Songqi Pan, Liushuan Dang, "On Energy Revolution and Science and Technology Revolution," Style of Journal of Southwest Petroleum University (Science \& Technology Edition), Vol. 41, No. 3, pp. 1-12, 2019.

[21] Erdong Zhao, Research on Low Carbonization Development of China's Electric Power Industry, Wuhan University, Wuhan, China, p. 127, 2012.

[22] Fuwei Guo "Development and Problems of New Energy," Shandong Industrial Technology, No. 19, pp. 230-231, 2019.

[23] Weiwei Feng, Songqi Pan, Liushuan Dang "Creating a Green Future Together to Provide New Ideas for Energy Conservation and Low Carbon Development," Energy Conservation \& Environmental Protection, No. 2, pp. 16-17, 2019. 